\title{
ON THE COMPUTATION OF EXCITED STATES WITH MCSCF METHODS
}

\author{
MATHIEU LEWIN
}

\begin{abstract}
We discuss the theoretical and practical problems arising when trying to compute excited states of nonrelativistic electrons in a molecular system, by multiconfiguration (MCSCF) methods. These nonlinear models approximate the linear Schrödinger theory and are a generalization of the well-known Hartree-Fock approach. Due to the MCSCF nonlinearity, a theoretical definition of what should be a MCSCF excited state is not clear at all, contrarily to the ground state case. We compare various definitions used in Quantum Chemistry. We in particular stress that some defects may lead to important computational problems, already observed in Quantum Chemistry (root flipping). We then present a definition of MCSCF excited states based on a solid mathematical ground and compare it with the most used methods. This new definition leads to a completely new algorithm for computing the first excited state, which was proposed and tested in a collaboration with Éric Cancès and Hervé Galicher. Numerical results are provided for the simple case of two-electron systems, as an illustration of the possible issues which can arise as consequences of the nonlinearity of the MCSCF method.
\end{abstract}

Electronic excited states play an essential role in various phenomena of high interest, such as photo-induced chemical reactions, femtosecond spectroscopy, or laser control of molecular processes. The method which seems to be best-adapted to the computation of excited states is to date the multiconfiguration self-consistent field (MCSCF) method. Loosely speaking, this approach leads to variational models which fill the gap between the mean-field Hartree-Fock and the $N$-body Schrödinger models. But important problems are encountered in practice during MCSCF computations for excited states. The algorithms available at present do not always converge (root-flipping problems) and, even when they converge, the interpretation of the obtained state is not always completely clear.

The goal of this paper is to provide some possible mathematical explanation of these phenomena. We shall in particular see that, due to the nonlinearity of the MCSCF models, the definition of what is an MCSCF excited state is not always clear. We shall present a mathematically valid definition which has been proposed by the author in [9], and compare it to the usual definitions used in Quantum Chemistry. Then, we describe a new algorithm for the computation of the first excited state which has been proposed and tested in a collaboration with Éric Cancès and Hervé Galicher in [2].

\section{The MCSCF Method}

The MCSCF method is a nonlinear approximate model for the computation of eigenfunctions and eigenvalues of the $N$-body Schrödinger Hamiltonian. For a

I would like to thank my collaborators Éric Cancès and Hervé Galicher with whom the results which are presented at the end of the paper were obtained [2]. I also would like to thank Patrick Cassam-Chenaï and Frédéric Patras for their kind invitation to the conference "Mathematical Methods for Ab Initio Quantum Chemistry" at Nice (FRANCE) in November 2005, where these results were presented. 
molecule containing $M$ pointwise nuclei of positive charges $z_{1}, \cdots, z_{M}$ and located at $\bar{x}_{1}, \cdots, \bar{x}_{M} \in \mathbb{R}^{3}$ (we work within the usual Born-Oppenheimer approximation), and $N$ nonrelativistic quantum electrons, the $N$-body Hamiltonian written in atomic units reads

$$
H_{N}=\sum_{i=1}^{N}\left(-\frac{1}{2} \Delta_{x_{i}}+V\left(x_{i}\right)\right)+\sum_{1 \leqslant i<j \leqslant N} \frac{1}{\left|x_{i}-x_{j}\right|} .
$$

It acts on normalized electronic wavefunctions $\Psi\left(x_{1}, \ldots, x_{N}\right) \in L_{a}^{2}\left(\left(\mathbb{R}^{3}\right)^{N}\right), \int_{\mathbb{R}^{3 N}} \Psi^{2}=$ 1. The subscript $a$ indicates that, due to the fermionic nature of the electrons, one solely considers wavefunctions $\Psi$ which are antisymmetric under permutations of variables:

$$
\Psi\left(x_{1}, \ldots, x_{i}, \ldots, x_{j}, \ldots, x_{N}\right)=-\Psi\left(x_{1}, \ldots, x_{j}, \ldots, x_{i}, \ldots, x_{N}\right) .
$$

Finally, $V$ is the electrostatic potential generated by the nuclei

$$
V(x)=-\sum_{m=1}^{M} \frac{z_{m}}{\left|x-\bar{x}_{m}\right|} \text {. }
$$

In what follows, we denote by $Z=\sum_{m=1}^{M} z_{m}$ the total nuclear charge. For the sake of simplicity, we do not take the spin into account in the first part of this paper, but the following arguments can be straightforwardly adapted to the case of spin-dependent wavefunctions.

When $Z>N-1$, it has been proved by Zhislin [18] and Zhislin-Sigalov [19], that the spectrum $\sigma\left(H_{N}\right)$ of $H_{N}$ has the form:

$$
\sigma\left(H_{N}\right)=\left\{\lambda_{0} \leq \lambda_{1} \leq \cdots \leq \lambda_{k} \leq \cdots\right\} \cup[\Sigma, \infty),
$$

where the $\left\{\lambda_{k}\right\}_{k \geq 0}$ are eigenvalues of finite multiplicity which are below and converge to the bottom of the essential spectrum $\Sigma$ as $k \rightarrow \infty$. A ground state is a normalized eigenfunction associated with the first eigenvalue $\lambda_{0}$, whereas excited states are obtained for $\lambda_{k}>\lambda_{0}$. A ground or excited state $\Psi_{k}$ is a solution of the time-independent Schrödinger equation $H_{N} \Psi_{k}=\lambda_{k} \Psi_{k}$. The $\left(\lambda_{k}\right)^{\prime} s$ can be obtained by the usual Rayleigh-Ritz formula

$$
\lambda_{k}=\inf _{\substack{V \subset \mathcal{D}\left(H_{N}\right) \\ \operatorname{dim}(V)=k+1}} \sup _{\substack{\Psi \in V, \int \Psi^{2}=1}}\left\langle\Psi\left|H_{N}\right| \Psi\right\rangle,
$$

where $\mathcal{D}\left(H_{N}\right)$ is the domain of $H_{N}$ and $\langle\cdot \cdot\rangle$ denotes the usual scalar product of $L^{2}\left(\mathbb{R}^{3 N}\right)$.

The MCSCF method is based on the following remark:

$$
L_{a}^{2}\left(\left(\mathbb{R}^{3}\right)^{N}\right)=\bigwedge_{n=1}^{N} L^{2}\left(\mathbb{R}^{3}\right)
$$

an equality which can be explicited in the following way. Consider an orthonormal basis $\left(\psi_{i}\right)_{1 \leq i}$ of $L^{2}\left(\mathbb{R}^{3}\right), \int_{\mathbb{R}^{3}} \psi_{i} \psi_{j}=\delta_{i j}$. Then, one obtains an orthonormal basis of $L_{a}^{2}\left(\left(\mathbb{R}^{3}\right)^{N}\right)$ by considering the antisymmetrized products $\left(\psi_{i_{1}} \wedge \cdots \wedge \psi_{i_{N}}\right)_{1 \leq i_{1}<\cdots<i_{N}}$ where $\psi_{i_{1}} \wedge \cdots \wedge \psi_{i_{N}}$ denotes the so-called Slater determinant of the $\psi_{i_{k}}$ 's:

$$
\left(\psi_{i_{1}} \wedge \cdots \wedge \psi_{i_{N}}\right)\left(x_{1}, \ldots, x_{N}\right)=\frac{1}{\sqrt{N !}} \operatorname{det}\left(\psi_{i_{k}}\left(x_{l}\right)\right)_{k, l} .
$$

In other words every antisymmetric wavefunction $\Psi$ is an infinite linear combination of such Slater determinants:

$$
\Psi=\sum_{1 \leq i_{1}<\cdots<i_{N}} c_{i_{1} \ldots i_{N}} \psi_{i_{1}} \wedge \cdots \wedge \psi_{i_{N}}
$$


the sum being convergent in $L_{a}^{2}\left(\left(\mathbb{R}^{3}\right)^{N}\right)$. Remark that $\int_{\mathbb{R}^{3 N}} \Psi^{2}=1$ is then equivalent to the condition $\sum_{i_{1}<\cdots<i_{N}}\left(c_{i_{1} \ldots i_{N}}\right)^{2}=1$.

In the MCSCF approximation, one restricts the number of Slater determinants appearing in (4), by restricting the number of occupied orbitals. Moreover, to obtain a suitable approximation, the orbitals will not be fixed but rather optimized. Let $K \geq N$ be the number of considered orbitals, denoted as $\varphi_{1}, \ldots, \varphi_{K} \in L^{2}\left(\mathbb{R}^{3}\right)$ and which still must satisfy the orthonormal condition $\int \varphi_{i} \varphi_{j}=\delta_{i j}$. Both the coefficients $\left(c_{i_{1}, \ldots, i_{N}}\right)_{1 \leq i_{k} \leq K}$ and the $K$ orbitals $\left(\varphi_{k}\right)_{k=1}^{K}$ are considered as being variational parameters, and the form of the $N$-body wavefunction $\Psi$ is now

$$
\Psi=\sum_{1 \leq i_{1}<\cdots<i_{N} \leq K} c_{i_{1} \ldots i_{N}} \varphi_{i_{1}} \wedge \cdots \wedge \varphi_{i_{N}}
$$

When there is no ambiguity, we shall use the following convenient notation

$$
\Psi=\sum_{I=\left\{i_{1}<\cdots<i_{N}\right\}} c_{I} \Phi_{I}
$$

where by definition $\Phi_{I}=\varphi_{i_{1}} \wedge \cdots \wedge \varphi_{i_{N}}$ when $I=\left\{i_{1}<\cdots<i_{N}\right\} \subset\{1, \ldots, K\}$.

Of course in practice not all the $\left(\begin{array}{l}K \\ N\end{array}\right)$ possible Slater determinants in (5) are considered during an MCSCF computation, and only some of them are selected. One of the most used method to choose an appropriate form for the wavefunction is the CASSCF method [12] in which the orbitals are divided into three groups (the active, inactive and virtual ones). We refer to [9] in which an appropriate formalism for treating these methods is provided. For the sake of clarity, we shall only consider in this proceeding the "full" MCSCF method (5) in which all orbitals are active.

When the quadratic form $\Psi \mapsto\left\langle\Psi\left|H_{N}\right| \Psi\right\rangle$ is restricted to states of the form (5), one obtains an energy depending on $\left(c_{i_{1}, \ldots, i_{N}}\right)_{1 \leq i_{k} \leq K}$ and $\left(\varphi_{1}, \ldots, \varphi_{K}\right) \in L^{2}\left(\mathbb{R}^{3}\right)^{K}$ which is nonlinear. This is due to the fact that functions of the form (5) do not form a vector subspace of $L_{a}^{2}\left(\left(\mathbb{R}^{3}\right)^{N}\right)$. In the following, we denote by $c=\left(c_{i_{1}, \ldots, i_{N}}\right)_{1 \leq i_{k} \leq K}$ the collection of the $c_{I}$ 's (lexicographical ordering), and by $\Phi=\left(\varphi_{1}, \ldots, \varphi_{K}\right) \in$ $\left(L^{2}\left(\mathbb{R}^{3}\right)\right)^{K}$ the collection of the orbitals. Due to the normalization constraint on the wavefunction $\Psi$, our variable $(c, \Phi)$ belongs to the following manifold

$$
\mathcal{M}_{N}^{K}=\left\{(c, \Phi) \in \mathbb{R}^{\left(\begin{array}{l}
K \\
N
\end{array}\right)} \times\left(L^{2}\left(\mathbb{R}^{3}\right)\right)^{K}, \sum_{I}\left(c_{I}\right)^{2}=1, \int_{\mathbb{R}^{3}} \varphi_{i} \varphi_{j}=\delta_{i j}\right\} .
$$

The MCSCF energy is then defined as $\mathcal{E}_{N}^{K}(c, \Phi)=\left\langle\Psi\left|H_{N}\right| \Psi\right\rangle$ where $\Psi$ is given by (5).

We shall not give here an explicit expression of the MCSCF energy in terms of $(c, \Phi)$ and refer to [9]. However, it is important to realize that the two variables $c$ and $\Phi$ play different roles. The energy $\mathcal{E}_{N}^{K}$ is still quadratic with respect to $c$ :

$$
\mathcal{E}_{N}^{K}(c, \Phi)=\sum_{I, J} c_{I} c_{J}\left\langle\Phi_{I}\left|H_{N}\right| \Phi_{J}\right\rangle=\sum_{I, J} c_{I} c_{J}\left(H_{\Phi}\right)_{I J}
$$

where $\left(H_{\Phi}\right)_{I J}=\left\langle\Phi_{I}\left|H_{N}\right| \Phi_{J}\right\rangle$ and $\Psi=\sum_{I} c_{I} \Phi_{I}$. The Hamiltonian matrix $H_{\Phi}$ is the $\left(\begin{array}{l}K \\ N\end{array}\right) \times\left(\begin{array}{l}K \\ N\end{array}\right)$ matrix of the quadratic form associated with $H_{N}$, when it is restricted to the $\left(\begin{array}{l}K \\ N\end{array}\right)$-dimensional space $V_{\Phi}=\operatorname{Span}\left(\Phi_{I}\right)$ spanned by the Slater determinants that can be constructed with the orbitals $\Phi=\left(\varphi_{1}, \ldots, \varphi_{K}\right)$. But, as mentioned above, the energy $\mathcal{E}_{N}^{K}$ is not quadratic with respect to the $\varphi_{i}$ 's and takes 
the following general form:

$$
\begin{aligned}
\mathcal{E}_{N}^{K}(c, \Phi)=\sum_{i, j} \frac{\gamma_{i j}}{2} \int_{\mathbb{R}^{3}}\left(\nabla \varphi_{i}\right. & \left.\cdot \nabla \varphi_{j}+V \varphi_{i} \varphi_{j}\right) \\
& +\sum_{i, j, k, l} W_{i j k l} \iint_{\mathbb{R}^{6}} \frac{\varphi_{i}(x) \varphi_{j}(x) \varphi_{k}(y) \varphi_{l}(y)}{|x-y|} d x d y
\end{aligned}
$$

where $\gamma_{i j}$ and $W_{i j k l}$ only depend on $c$. The first sum in (7) is quadratic whereas the last term is quartic. Thus, the MCSCF equations (i.e. the equations satisfied by a stationary point of $\mathcal{E}_{N}^{K}$ on the manifold $\mathcal{M}_{N}^{K}$ ) consist in an eigenvalue equation for $c$ coupled with a system of $K$ nonlinear elliptic partial differential equations for $\left(\varphi_{1}, \ldots, \varphi_{K}\right)$, of the general form

$$
\left\{\begin{array}{l}
n_{i}(c)\left(-\frac{\Delta}{2}+V\right) \varphi_{i}+\sum_{j} W_{i, j}^{(c, \Phi)} \varphi_{j}=\sum_{j} \lambda_{i j} \varphi_{j} \\
H_{\Phi} c=\beta c .
\end{array}\right.
$$

The operators $W_{i, j}^{(c, \Phi)}$ depend on $(c, \Phi)$ in a complicated manner.

Mathematically, it is not at all obvious to prove the existence of solutions to the MCSCF equations (8) when the problem is posed in infinite dimension. There is a possible lack of compactness at infinity: for any sequence $\left(c_{n}, \Phi_{n}\right)$ of approximate solutions of (8) it is possible that some electrons "escape to infinity" as $n \rightarrow \infty$ [4]. This phenomenon will typically occur when $N \gg Z$, if the nuclei are not able to bind the $N$ electrons.

Of course, in practice the computation is carried out in finite dimension: the space $L^{2}\left(\mathbb{R}^{3}\right)$ is replaced by a finite dimensional space $V$, usually spanned by a chosen finite set of atomic orbitals. Then the possible lack of compactness mentioned above never occurs. Notice however that the problems that will be raised below concerning the existence and the computation of excited states (specific solutions of (8) which approximate the true eigenfunctions of $H_{N}$ ) appear in finite dimension also. They will not be related to the mathematical problem of lack of compactness at infinity which only occurs in infinite dimension.

The definition of the MCSCF ground state energy is clear: it suffices to minimize the energy $\mathcal{E}_{N}^{K}$ on $\mathcal{M}_{N}^{K}$, i.e. to minimize the $N$-body energy over MCSCF functions of the type (5):

$$
\lambda_{0}^{K}=\inf _{(c, \Phi) \in \mathcal{M}_{N}^{K}} \mathcal{E}_{N}^{K}(c, \Phi) .
$$

It can be proved that $\lambda_{0}^{K}>\lambda_{0}$ and that $\lim _{K \rightarrow \infty} \lambda_{0}^{K}=\lambda_{0}$, which justifies the MCSCF approach for ground states. Recall that $K$ is the number of orbitals appearing in the model.

In infinite dimension, the existence of an MCSCF ground state was proved when $Z>N-1$ first by C. Le Bris [8] for a subcase of $K=N+2$, and then by G. Friesecke [4] in the general case $K \geq N$. In the latter article, it is also shown that the MCSCF ground state wavefunction $\Psi_{0}^{K}$ converges to the true eigenfunction $\Psi_{0}$ of $H_{N}$. Finally, the case of the methods used in practice like the CASSCF method was studied by the author in [9].

Numerically, a minimizer of (9) is usually computed by a Newton-like algorithm, sometimes improved by a trust-region method, see e.g. [14, 15, 13, 3, 5, 6] and the references in [2]. For the Hartree-Fock model $K=N$, efficient numerical methods based on combinations of fixed-point and optimization strategies are available [1]. Unfortunately, such algorithms are specifically designed for solving the Hartree-Fock problem and seem to be difficult to adapt to the more general MCSCF setting. 


\section{MCSCF EXCiTEd STATES}

It is not obvious at all to define what an MCSCF excited state is. Indeed, due to the nonlinearity, the functional $\mathcal{E}_{N}^{K}$ has many spurious stationary points. In [2], the following general guiding principles for a $d^{\text {th }}$ excited state were proposed:

- P1 (First order condition) It should be a critical point of $\mathcal{E}_{N}^{K}$ on $\mathcal{M}_{N}^{K}$, i.e. a solution of the MCSCF equations (8).

- P2 (Second order condition) Its total Hessian matrix should have at most d negative eigenvalues [5]. This in particular implies that $c$ should be at most the $(d+1)^{\text {th }}$ eigenvector of the Hamiltonian matrix $H_{\Phi}$. But in principle, it could be a lower eigenvector, because the $d$ negative directions of the Hessian matrix do not necessarily correspond to variations of $c$ only.

- P3 (HUM-type theorem and large $K$ behavior) Its energy $\lambda_{d}^{K}$ should satisfy $\lambda_{d}^{K} \geq \lambda_{d}$ and $\lim _{K \rightarrow \infty} \lambda_{d}^{K}=\lambda_{d}$. The associated wavefunction $\Psi_{d}^{K}$ should converge to the true excited state $\Psi_{d}$, solution of $H_{N} \Psi_{d}=\lambda_{d} \Psi_{d}$.

If one wants to impose the third property $\mathbf{P 3}$, there is a natural definition for the MCSCF excited state energies, which is indeed the one which is mostly used in Quantum Chemistry (see, e.g. [13, 14, 15] and the references of [2]). Let us denote by $\mu_{d}^{K}(\Phi)$ the $\left(\begin{array}{c}K \\ N\end{array}\right)$ eigenvalues of the Hamiltonian matrix $H_{\Phi}$, depending on the orbitals $\Phi=\left(\varphi_{1}, \ldots, \varphi_{K}\right)$. By the usual Rayleigh-Ritz formula (2), one deduces that

$$
\lambda_{d} \leq \mu_{d}^{K}(\Phi) .
$$

This inequality is usually referred to as the Hylleraas-Undheim-MacDonald (HUM) theorem in Chemistry. It is therefore natural to define the following energy:

$$
\mu_{d}^{K}=\inf _{\Phi} \mu_{d}^{K}(\Phi)
$$

that is to say, quoting [13], "the MCSCF energy results from minimizing the appropriate eigenvalue of the Hamiltonian matrix with respect to orbital variations". It can actually be proved that $\mu_{d}^{K} \searrow \lambda_{d}$ as $K \rightarrow \infty$. However, (10) is a minimization of an eigenvalue of a symmetric matrix depending on a parameter $\Phi$. This type of variational method is generally very ill-posed mathematically (even in finite dimensions) and we believe that most of the convergence problems encountered in practical computations are due to this issue. We shall now give very simple examples in dimensions 1 or 2 to illustrate the possible theoretical and numerical difficulties when trying to optimize the eigenvalue of a matrix depending on a parameter.

First, it can happen that there is no stationary state $(c, \Phi)$ solution of (8) with an energy $\mu_{d}^{K}$, in which case it will not be possible to find a state satisfying the properties $\mathbf{P 1}$ and $\mathbf{P 2}$. We give as an example an energy of the form $E(v, \mathbf{x})=$ $(v, A(\mathbf{x}) v)_{\mathbb{R}^{2}}$ where $v$ is a normalized vector in $\mathbb{R}^{2}, A(\mathbf{x})$ is a symmetric matrix depending on the parameter $\mathbf{x} \in \mathbb{R}^{2}$, and $(\cdot, \cdot)_{\mathbb{R}^{2}}$ denotes the usual scalar product of $\mathbb{R}^{2}$. In our situation, $\mathbf{x}$ corresponds to the set of the orbitals $\Phi$ whereas $v$ plays the role of $c$. Let us consider the following matrix

$$
A(\mathbf{x})=\left(\begin{array}{cc}
-\sin x & \sin y \\
\sin y & \sin x
\end{array}\right),
$$

where $\mathbf{x}=(x, y) \in \mathbb{R}^{2}$. The two eigenvalues of $A(\mathbf{x})$ are

$$
\mu_{1}(\mathbf{x})=-\sqrt{x^{2}+y^{2}} \text { and } \mu_{2}(\mathbf{x})=\sqrt{x^{2}+y^{2}},
$$

see Figure 1. Hence

$$
\inf _{\mathbf{x} \in \mathbb{R}^{2}} \mu_{2}(\mathbf{x})=0
$$




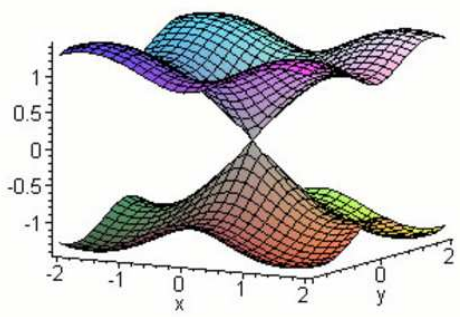

Figure 1. The eigenvalues of the matrix $A(\mathbf{x})$

the infimum being attained when $\mathbf{x}=0$. But it is an exercise to check that the energy $(v, \mathbf{x}) \mapsto(v, A(\mathbf{x}) v)_{\mathbb{R}^{2}}$ has no stationary state of the form $(v, 0)$.

A second important problem is that there is no general well-behaved numerical method for minimizing the eigenvalue of a symmetric matrix depending on a parameter, except in very special cases not applicable to our situation. One might be temped to consider the following naive two-step algorithm, in which the orbitals and the coefficients $c_{I}$ 's are optimized separately:

\section{Algorithm 1.}

(1) $\Phi$ being fixed, take $c^{\prime}$ to be a $(d+1)^{\text {th }}$ eigenvector of $H_{\Phi}$;

(2) $c^{\prime}$ being fixed, find $\Phi^{\prime}$ as a minimizer of $\tilde{\Phi} \mapsto \mathcal{E}_{N}^{K}\left(c^{\prime}, \tilde{\Phi}\right)$ and go back to (1).

This naive algorithm does not always converge and can oscillate between non physical states. As before, we give an example for a toy model in finite dimension. We introduce $E(v, x)=(v, B(x) v)_{\mathbb{R}^{2}}$ where $v$ is a normalized vector in $\mathbb{R}^{2}, x \in \mathbb{R}$ and $B(x)$ is this time defined as:

$$
B(x)=\left(\begin{array}{cc}
-\sin x & 0 \\
0 & \sin x
\end{array}\right) .
$$

When Algorithm 1 is adapted to this example, one obtains an oscillation between $x=-\pi / 2$ and $x=\pi / 2$.

In many Quantum Chemistry programs, an improved version of Algorithm 1 is implemented $[13,16,14]$. The oscillation phenomenon reported on in the previous example is called root-flipping and is a typical drawback when trying to compute MCSCF excited states. Many solutions have been proposed to avoid this issue. First, the computation is always done in a special symmetry subspace in order to avoid degeneracy problems. Then, it has been proposed to optimize the average of different eigenvalues $\mu_{d}^{K}$ 's instead of only one [16]. On example (12), the optimization of $\sin (x) / 2-\sin (x) / 2=0$ of course leads to the correct answer, but this is the only convex combination of the two eigenvalues that solves the root-flipping problem. Any other combination of the form $m \sin (x)+(1-m)(-\sin (x))=(2 m-1) \sin (x)$ with $m \in[0 ; 1], m \neq 1 / 2$, leads to the same root-flipping phenomenon.

Even when there is a stationary state with an energy $\mu_{d}^{K}$, and when no rootflipping is encountered, the state obtained with Definition (10) can be unphysical. An example of such a situation is given in [10] for a two-electron system. This will be developed below in Section 3. All this means that (10) cannot be considered as a relevant definition in general: imposing that $c$ is a specific eigenvector of $H_{\Phi}$ may lead to unphysical results. 
The issues raised by Definition (10) have already been described and studied in details in $[7,5,17,11]$ by the team of the DALTON software [20]. They proposed a different definition of excited states which consists in just imposing that only the first two properties $\mathbf{P 1}$ and $\mathbf{P 2}$ hold. A $d^{\text {th }}$ excited state is therefore defined as a stationary state whose total Hessian matrix has exactly $d$ negative eigenvalues. Such states are computed in DALTON by a well-behaved Newton-type algorithm followed by a trust region method, which does not lead to any root-flipping problem. However, one might ask why any stationary state whose Hessian matrix has the right number of negative eigenvalues would correspond to an approximation of the true excited state. Recall that due to the nonlinearity, many spurious stationary states may exist. We will provide below an example of an MCSCF solution whose Hessian matrix has exactly one negative eigenvalue and which is not at all an approximation of the first excited state. Finally, states which do not satisfy the property $\mathbf{P 3}$ can be obtained by the DALTON method ${ }^{1}$, which is not completely satisfactory.

In [9], an alternate definition of MCSCF excited state energies was proposed and the following theorem was proved:

Theorem 1 (Existence of MCSCF Excited States [9]). Let be $Z>N-1$. For any $K \geq N$ and any $d=0, \ldots,\left(\begin{array}{l}K \\ N\end{array}\right)-1$, there exists a stationary state $\left(c_{d}^{K}, \Phi_{d}^{K}\right)$ of $\mathcal{E}_{N}^{K}$ on $\mathcal{M}_{N}^{K}$, whose Hessian matrix has at most $d$ negative eigenvalues, and which is such that, denoting $\lambda_{d}^{K}=\mathcal{E}_{N}^{K}\left(c_{d}^{K}, \Phi_{d}^{K}\right)$,

$$
\lambda_{d} \leq \lambda_{d}^{K} \leq \mu_{d}^{K}, \quad \text { and } \lim _{K \rightarrow \infty} \lambda_{d}^{K}=\lambda_{d} .
$$

Except the convergence of the wavefunction as $K \rightarrow \infty$ which is still not proved mathematically, one can therefore construct an MCSCF excited state which satisfies the three properties P1-P3. In Theorem 1, it is not explained how the MCSCF excited states are defined and constructed. Indeed, they are obtained by complicated min-max variational methods which fully take into account the nonlinearity of the functional $\mathcal{E}_{N}^{K}$, and differ from (10). Unfortunately, these variational methods are quite complicated to present and also probably too involved to be used in practice when $d$ is too large. For this reason, we shall now only present and explain the case of the first excited state energy $\lambda_{1}^{K}$. Details for higher excited states can be found in $[9,2]$.

As explained in [2], the variational formula providing the first excited state $\left(c_{1}^{K}, \Phi_{1}^{K}\right)$ of Theorem 1 is the following:

$$
\lambda_{1}^{K}=\inf _{(c, \Phi) \in \mathcal{M}_{N}^{K}}\left\{\inf _{\gamma \in \Gamma_{(c, \Phi)}} \sup _{t \in[0 ; 1]} \mathcal{E}_{N}^{K}(\gamma(t))\right\}
$$

where

$$
\Gamma_{(c, \Phi)}=\left\{\gamma \in C^{0}\left([0 ; 1], \mathcal{M}_{N}^{K}\right), \gamma(0)=(c, \Phi), \gamma(1)=(-c, \Phi)\right\} .
$$

It was argued in [2] that a solution of the first inf in (13) is very probably given by an MCSCF ground state $\left(c_{0}, \Phi_{0}\right)$, i.e. such that $\mathcal{E}_{N}^{K}\left(c_{0}, \Phi_{0}\right)=\lambda_{0}^{K}$ (we do not write the superscript $K$ on $\left(c_{0}, \Phi_{0}\right)$ for simplicity). In this case, (13) reduces to

$$
\lambda_{1}^{K}=\inf _{\substack{\gamma:[0 ; 1] \rightarrow \mathcal{M}_{N}^{K} \\ \gamma(0)=\left(c_{0}, \Phi_{0}\right), \gamma(1)=\left(-c_{0}, \Phi_{0}\right)}} \sup _{t \in[0 ; 1]} \mathcal{E}_{N}^{K}(\gamma(t)) .
$$

${ }^{1}$ H.J.Aa. Jensen, private communication. 


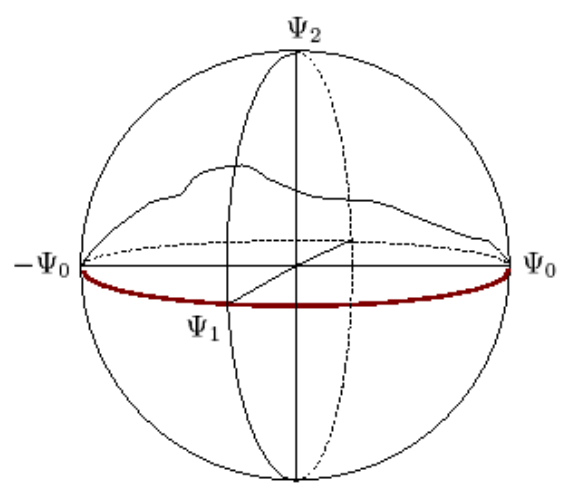

Figure 2. The true (Schrödinger) first excited state $\Psi_{1}$ can be obtained as a mountain pass point between the two ground states $\pm \Psi_{0}$ : any path linking $\Psi_{0}$ and $-\Psi_{0}$ necessarily intersects the orthogonal of $\operatorname{Span} \Psi_{0}$, on which the energy is $\geq \lambda_{1}$.

This formula is probably clearer to the reader: it corresponds to a mountain pass problem between the two ground states $\left(c_{0}, \Phi_{0}\right)$ and $\left(-c_{0}, \Phi_{0}\right)$, similar to those encountered in molecular simulation in the search for transition states between reactants and products on potential energy surfaces. The function $\gamma$ in (14) represents the path, parametrized on $[0 ; 1]$. The path which has the smallest higher energy is the one which goes through the mountain pass point, the latter being interpreted as the first MCSCF excited state.

Let us now explain why such a mountain pass problem to find the first nonlinear excited state is somehow natural. Let us denote by $\bar{\Psi}_{0}$ the MCSCF ground state wavefunction associated with $\left(c_{0}, \Phi_{0}\right)$, which is a good approximation of the true Schrödinger ground state $\Psi_{0}$ when $K$ is large enough. Then the wavefunction associated with $\left(-c_{0}, \Phi_{0}\right)$ is $-\bar{\Psi}_{0}$ and (14) corresponds to a mountain pass problem between $\pm \bar{\Psi}_{0}$, on the MCSCF manifold. This is now very natural since the true first excited state $\Psi_{1}$ can also be obtained as a mountain pass point between $\pm \Psi_{0}$, but on the whole sphere of $L_{a}^{2}\left(\mathbb{R}^{3 N}\right)$ (not only MCSCF states). An optimal path is given by the half circle passing through $\Psi_{0}$ and $\Psi_{1}$, see Figure 2 .

Therefore, the computation of the first excited state is reduced to the problem of finding a transition state between two ground states, on the manifold $\mathcal{M}_{N}^{K}$. This gives rise to the following new algorithm which was proposed, studied and tested in $[2]$ :

\section{Algorithm 2.}

(1) Find an MCSCF ground state $\left(c_{0}, \Phi_{0}\right)$, by a Newton-like method.

(2) Find an initial path $\gamma_{0}$ linking $\left(c_{0}, \Phi_{0}\right)$ and $\left(-c_{0}, \Phi_{0}\right)$ on the manifold $\mathcal{M}_{N}^{K}$. For instance one can choose the following half circle where the orbitals are frozen: $\gamma_{0}(t)=\left(c(t), \Phi_{0}\right)$ with $c(t)=\cos (\pi t) c_{0}+\sin (\pi t) c_{0}^{\prime}$ where $c_{0}^{\prime}$ is the second eigenvector of the Hamiltonian matrix $H_{\Phi_{0}}$. This initial path can then be perturbed randomly.

(3) Deform $\gamma_{0}$ to solve the mountain pass problem between $\left(c_{0}, \Phi_{0}\right)$ and $\left(-c_{0}, \Phi_{0}\right)$.

(4) When the highest point on the path has a small-enough derivative, switch to a Newton algorithm.

We refer to [2] for a detailed explanation of the method which has been used to deform the paths, and which could be useful in other setting, for instance when one wants to compute a transition state during a chemical reaction. 
Remark: on the orthogonality of the MCSCF wavefunctions. It is important to realize that the wavefunction $\bar{\Psi}_{1}$ of the first MCSCF excited state $\left(c_{1}, \Phi_{1}\right)$ is not necessarily orthogonal to the MCSCF ground state wavefunction $\bar{\Psi}_{0}$. In some Quantum Chemistry programs, the orthogonality between the ground and the first excited states is always assumed. This is also a reason why an average of the first two eigenvalues of the Hamiltonian matrix is sometimes optimized: the computation aims at finding both the MCSCF ground state and the first MCSCF excited state, in the same orbital basis $\left(\varphi_{1}, \ldots, \varphi_{K}\right)$.

However, there is no reason for assuming that the MCSCF ground state and the first MCSCF excited state are orthogonal: this is a linear property, true for the Schrödinger linear model, and which will not necessarily be true for the MCSCF nonlinear approximation. Imposing this condition might lead to important theoretical and numerical problems, similar to the ones encountered and explained above. Indeed, it is the (nonlinear) mountain pass configuration which replaces the orthogonality property in the case of the MCSCF first excited state. Of course, in the limit $K \rightarrow \infty$, the states will become orthogonal and there will be a choice for the optimal path which converges to the half circle. We notice that in DALTON [20], different excited states are also not necessarily orthogonal.

\section{A TEST CASE: TWO-ELECTRON SYSTEMS}

In this last section, we report some numerical results that have been obtained in [2], using Algorithm 2, on the simple case of the singlet state of two-electron systems. Interesting properties in relation with previous remarks will be demonstrated when the MCSCF wavefunction is not assumed to belong to any specific symmetry subspace.

We shall concentrate on the $\mathrm{H}_{2}$ molecule. We assume that the two protons are located at $(-R / 2,0,0)$ and $(R / 2,0,0)$ in $\mathbb{R}^{3}$. We restrict ourselves to singlet states which take the form

$$
\Psi\left(x, \sigma ; y, \sigma^{\prime}\right)=\psi(x, y)|\alpha \beta\rangle\left(\sigma, \sigma^{\prime}\right)
$$

where $\psi$ is symmetric, $\psi(x, y)=\psi(y, x)$, i.e. the antisymmetry is in the spin variable. Of course, all the previous study can be easily adapted to the case of symmetric two-body wavefunctions.

The Schrödinger Hamiltonian (denoted as $H$ in this section) commutes with the symmetry operator $\tau$ defined as $(\tau f)(x, y)=f(-x,-y)$, i.e. $H \tau=\tau H$. Therefore $H$ stabilizes the two eigenspaces of $\tau$ which are the spaces of even and odd two-body wavefunctions

$$
\Sigma_{g}:=\{\psi \mid \psi(-x,-y)=\psi(x, y)\} \quad \text { and } \quad \Sigma_{u}:=\{\psi \mid \psi(-x,-y)=-\psi(x, y)\} .
$$

It is known in Chemistry that the ground state is in the $\Sigma_{g}$ symmetry whereas the first excited state is in the $\Sigma_{u}$ symmetry. It is therefore natural to find the first excited state as a minimizer in the $\Sigma_{u}$ subspace, and this is what is done in the usual Quantum Chemistry programs.

However, the following will be dedicated to the behavior of the MCSCF method in the full space, without taking any space symmetry into account. Of course, we do not claim at all that this should be used in practice to compute the first excited state! But rather this example provides a nice illustration of the possible difficulties: it could be useful for a better understanding of the behavior of the MCSCF method when it is used for the computation of excited states which are not the lowest of their symmetry.

Algorithm 2 was applied to find the first singlet excited state of the $\mathrm{H}_{2}$ molecule, in the cc-pVDZ atomic basis of orbitals. In Figure 3, the energy along the successive paths generated by the algorithm is shown. The MCSCF first excited state is the 


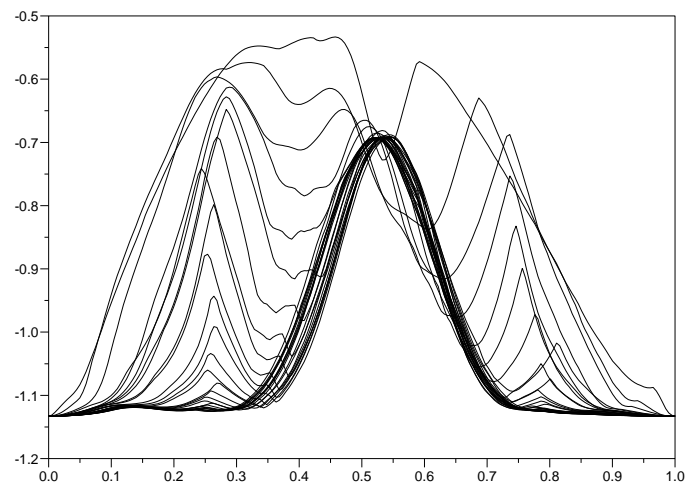

FIGURE 3. Energy along the successive deformed paths generated by Algorithm 2 for the computation of the first singlet excited state of $\mathrm{H}_{2}$, with $R=1 \AA$.

state of highest energy on the converged path, and it has two interesting features. First, its parameter $c$ is the first eigenvector of the Hamiltonian matrix $H_{\Phi}$, and not the second as this is imposed by (10). This was predicted first by McCourt and McIver in [10]. Notice however that the obtained energy is known to be an upper bound to the true Schrödinger first excited state energy by Theorem 1, but this has nothing to do with the usual linear Hylleraas-Undheim-MacDonald Theorem.

Then, our first excited state is close to the one which is computed by the usual chemistry programs in the $\Sigma_{u}$ symmetry, but not equal to it. Indeed, it is also only very close to be in the $\Sigma_{u}$ symmetry subspace, but does not exactly belong to $\Sigma_{u}$. On the other hand it has a very small gradient (of the order of $10^{-8}$ ), whereas the gradient of the state found by restriction to the $\Sigma_{u}$ space has a norm only of order $10^{-4}$ (of course when all variations into account, and not only those of the $\Sigma_{u}$ symmetry). This shows that the restriction to the $\Sigma_{u}$ subspace is not necessarily compatible with a nonlinear method like MCSCF: the MCSCF excited states do not a priori satisfy the symmetries of the Schrödinger solution. This kind of symmetry breaking is very usual for nonlinear theories. On the other hand, notice that there is no symmetry breaking for the MCSCF ground state: even when there is no symmetry constraint, the MCSCF ground state is indeed automatically found in the $\Sigma_{g}$ subspace.

Since there is no degeneracy problem, it was possible in [2] to find a solution of the eigenvalue minimization problem (10), which is the one used in most Quantum Chemistry programs $\mu_{1}^{K}$. What is surprising is that the so-obtained state does not at all approximate the first excited state: its energy is greater than the one computed by the mountain pass method, $\mu_{1}^{K}>\lambda_{1}^{K}$, and the optimal state itself belongs to the $\Sigma_{g}$ symmetry subspace. This shows that imposing that $c$ is a specific eigenvector of the Hamiltonian matrix $H_{\Phi}$ might lead to a spurious solution, although the Hessian matrix has exactly one negative eigenvalue. This also shows that not all the stationary states having this last property are related to the true first excited state.

The above computation were carried out in [2] for different values of the distance $R$, giving the Potential Energy Surface of the singlet state of $\mathrm{H}_{2}$, see Figure 4 . Notice that the spurious solution of (10) has an energy which is closer to the true 


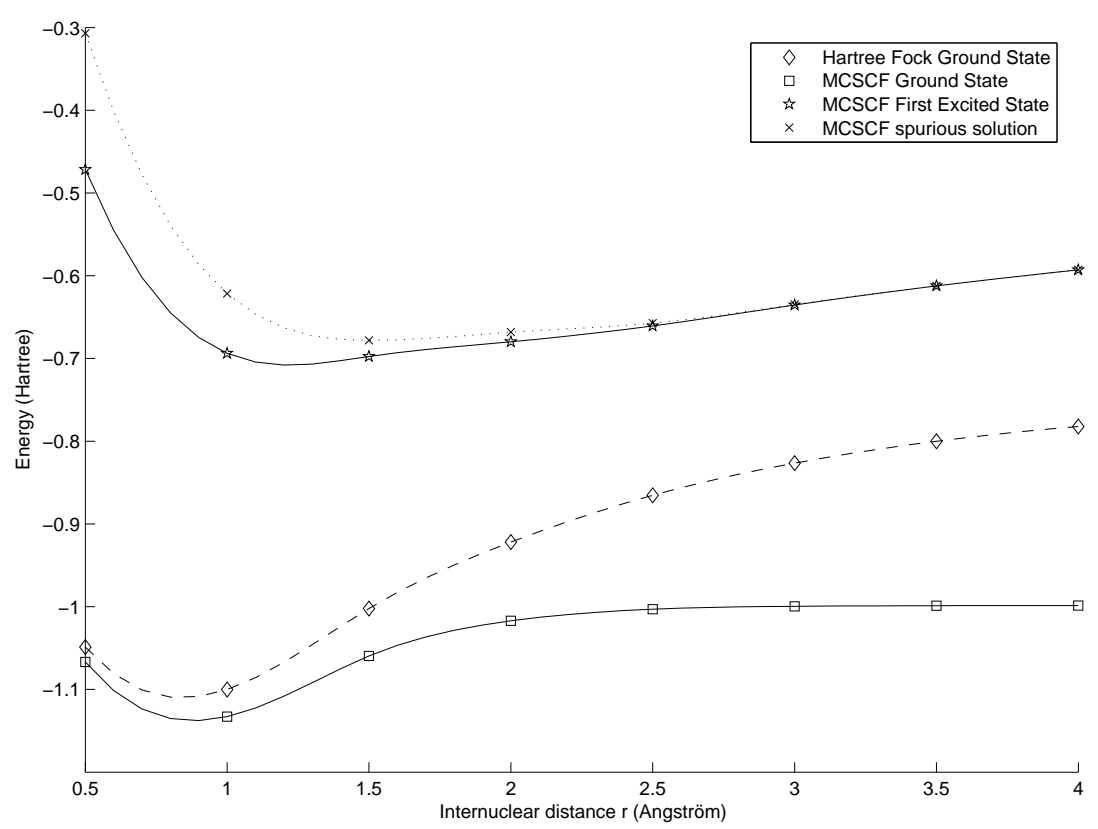

Figure 4. Potential Energy Surface of the singlet state of the molecule $\mathrm{H}_{2}$.

energy as $R$ grows, but the state itself is far from the true solution since it belongs to the $\Sigma_{g}$ subspace.

As a conclusion, we have shown that the nonlinear features of the MCSCF model raise important issues for the definition and the computation of excited states. Some properties which are true for the linear Schrödinger model (orthogonality, symmetries...) can be lost when using the MCSCF approximation. Imposing them by brute force may sometimes lead to wrong results, or to involved numerical difficulties. A mathematically well-posed definition was provided in [9], but its complicated formulation (unavoidable consequence of the MCSCF nonlinearity) probably restricts its applicability to the first or possibly the second excited states only. For the first excited state, it consists in solving a mountain pass between two MCSCF ground states whose configuration coefficients $c$ are opposite. This method could be used to compute first excited states which are not the lowest of their symmetry, and for which the usual methods might fail. It could help in understanding better the behavior of the nonlinear MCSCF methods.

\section{REFERENCES}

[1] E. Cancès, M. Defranceschi, W. Kutzelnigg, C. Le Bris and Y. Maday, Computational quantum chemistry: a primer, Handbook of Numerical Analysis vol. X, Elsevier, Amsterdam (2003), 3-270.

[2] É. Cancès, H. Galicher \& M. Lewin. Computing electronic structures : a new multiconfiguration approach for excited states. J. Comput. Phys. 212 (2006), 73-98.

[3] R. Eade and M. Robb, Direct minimization in MCSCF theory. The quasi-Newton method, Chem. Phys. Lett. 83 (1981), no. 2, 362-368.

[4] G. Friesecke, The multiconfiguration equations for atoms and molecules: charge quantization and existence of solutions, Arch. Rat. Mech. Anal. 169 (2003), 35-71. 
[5] P. Jørgensen, J. Olsen and D. Yeager, Generalizations of Newton-Raphson and multiplicity independent Newton-Raphson approaches in multiconfigurational Hartree-Fock theory, J. Chem. Phys. 75 (1981), no. 12, 5802-5815.

[6] P. Jørgensen, P. Swanstrøm and D. Yeager, Guaranteed convergence in ground state multiconfigurational self-consistent field calculations, J. Chem. Phys. 78 (1983), no. 1, 347-356.

[7] J. Golab, D. Yeager and P. Jørgensen. Multiple stationary point representation in MCSCF calculations, Chem. Phys. 93 (1985), 83-100.

[8] C. Le Bris, A general approach for multiconfiguration methods in quantum molecular chemistry, Ann. Inst. H. Poincaré Anal. Non linéaire 11 (1994), no. 6, 441-484.

[9] M. Lewin. Solutions of the multiconfiguration equations in quantum chemistry. Arch. Ration. Mech. Anal. 171 (2004), no. 1, 83-114.

[10] M. McCourt and J. McIver Jr. On the SCF calculation of excited states: singlet states in the two-electron problem, J. Comput. Chem. 8 (1987), no. 4, 454-458

[11] J. Olsen, P. Jørgensen and D. Yeager, Multiconfigurational Hartree-Fock studies of avoided curve crossing using the Newton-Raphson technique, J. Chem. Phys. 76 (1982), no. 1, 527542 .

[12] B.O. Roos, The complete active space self-consistent field method and its applications in electronic structure calculation. Ab initio methods in quantum chemistry - II, Adv. Chem. Phys. 69 (1987), 399-446.

[13] R. Shepard, The multiconfiguration self-consistent field method. Ab initio methods in quantum chemistry - II, Adv. Chem. Phys. 69 (1987), 63-200.

[14] H.-J. Werner, Matrix-formulated direct multiconfiguration self-consistent field and multiconfiguration reference Configuration-Interaction methods. Ab initio methods in quantum chemistry - II, Adv. Chem. Phys. 69 (1987), 1-62.

[15] H.-J. Werner and P. Knowles, A second order multiconfiguration SCF procedure with optimum convergence, J. Chem. Phys. 82 (1985), no. 11, 5053-5063.

[16] H.-J. Werner and W. Meyer, A quadratically convergent MCSCF method for the simultaneous optimization of several states, J. Chem. Phys. 74 (1981), no. 10, 5794-5801.

[17] D. Yeager, D. Lynch, J. Nichols, P. Jørgensen and J. Olsen, Newton-Raphson approaches and generalizations in multiconfigurational self-consistent field calculations, J. Phys. Chem. 86 (1982), 2140-2153

[18] G. M. Zhislin. A study of the spectrum of the Schrödinger operator for a system of several particles. (Russian) Trudy Moskov. Mat. Obšč. 9 (1960), 81-120.

[19] G. M. Zhislin and A.G. Sigalov. The spectrum of the energy operator for atoms with fixed nuclei on subspaces corresponding to irreducible representations of the group of permutations. (Russian) Izv. Akad. Nauk SSSR Ser. Mat. 29 (1965), 835-860. Transl. A.M.S. Ser. 2 91, 263-296 (English translation).

[20] DALTON, a molecular electronic structure program. See http://www.kjemi.uio.no/software/dalton/dalton.html.

CNRS \& Département de Mathématiques, Université de Cergy-Pontoise, 2 avenue Adolphe Chauvin, 95302 Cergy-Pontoise Cedex, FRANCE.

E-mail address: Mathieu.Lewin@math.cnrs.fr 